\title{
The Energy Calibration Using the Moon Shadow of LHAASO-WCDA Detector
}

\author{
Yanjin Wang ${ }^{a b}$ Min Zha ${ }^{b}$, Zhen $\mathrm{Cao}^{b}$, Xin Zhang ${ }^{a}$ for the LHAASO Collaboration \\ ${ }^{a}$ Northeastern University, Shenyang 110004, China. \\ ${ }^{b}$ Institute of High Energy Physics, Chinese Academy of Sciences, Beijing, 100049, China. \\ E-mail: wangyanjin1992@126.com \\ E-mail: Min Zhadihep.ac.cn \\ E-mail: caozhdihep.ac.cn \\ E-mail: Zhangxinemail.neu.edu.cn
}

\begin{abstract}
The shadow of the Moon observed with cosmic rays can be utilized to study the pointing error and the angular resolution of the ground-based air shower arrays, also offers a good ability to do below a few $\mathrm{TeV}$ energy calibration based on the bending of cosmic rays when they travel through the geomagnetic field. Water Cherenkov Detector Array (WCDA), one of components of Large High Altitude Air Shower Observation (LHAASO), has high observation sensitivity, lower threshold, and high duty cycle, which make itself a very good facility in this aspect. In this presentation, we study the ability of the westward displacement of the Moon shadow based on an accurate simulation and experimental data analysis, some systematic uncertainties related with energy calibration are also discussed.
\end{abstract}

36th International Cosmic Ray Conference -ICRC2019-

July 24th - August 1st, 2019

Madison, WI, U.S.A. 


\section{Introduction}

The shadow of the moon is formed by that the cosmic ray transmission to earth is hampered by the moon. Much useful information can be provided by the moon shadow. For example, the study of the moon shadow can not only check the pointing error of detector, estimate the angular resolution of the detector, calculate the $\bar{p} / \mathrm{p}$ ratio, but can also discuss the ability of detector on energy calibration [[]]. In one word the Earth-Moon system can be treated as a spectrometer, based on the method of Monte Carlo simulation, a link between shower size and primary energy could be established.

The CYGNUS experiment [3] firstly observed the effect of moon shadow and sun shadow in 1991 , and they got the $4.9 \sigma$ significance of moon shadow. But they don't observe the deflection of moon shadow because of the high threshold of the detector. Using the high altitude and low threshold energy features of LHAASO-WCDA, we are going to select the moon shadow data by using light components during+6 the low energy and to establish the energy calibration.

This paper is organized as follows. The LHAASO-WCDA detector is introduced in Section 2. Detailed simulation of moon shadow is provided in Section 3. The moon shadow of WCDA data is analyzed in Section 4. In Section 5, moon shadow results of simulation and WCDA data test each other and a short future outlook is presented.

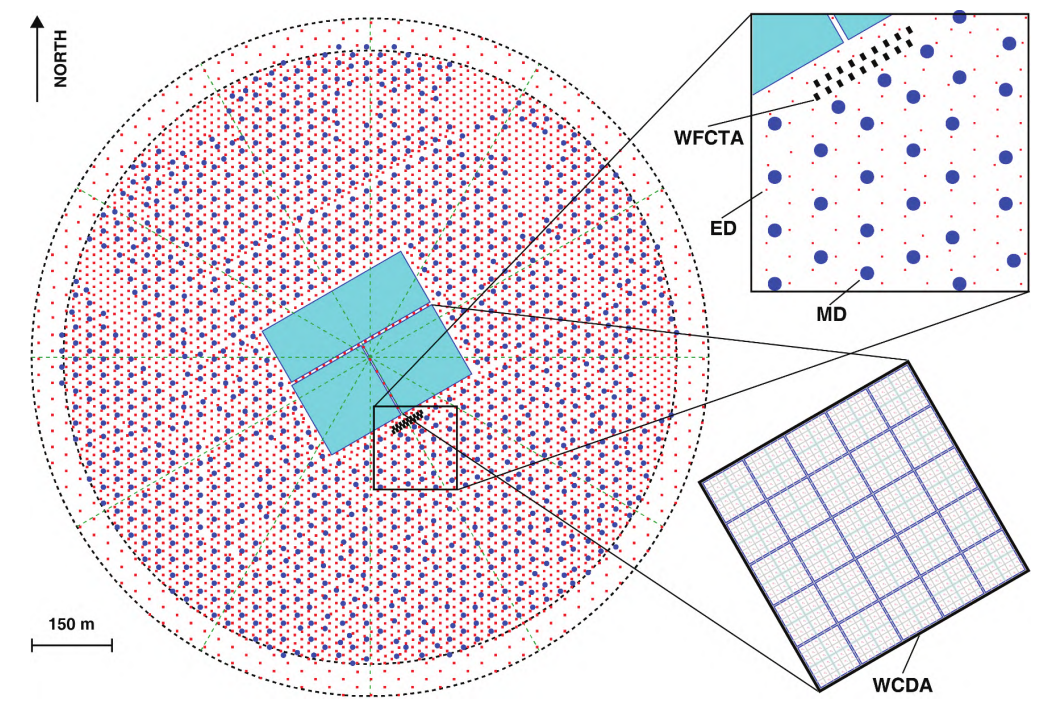

Figure 1: The layout of LHAASO detectors [四].

\section{The LHAASO-WCDA detector}

The Large High Altitude Air Shower Observation(LHAASO) is located in Mountain Haizishan(altitude, 4410m asl) Daocheng, Sichuan Province, China, which consists of Kilometer-square Array (KM2A), Water Cherenkov Detector Array (WCDA), Wide Field of View Cherenkov Telescope Array (WFCTA). LHAASO has advantages such as high sensitivity and powerful sky survey ability, which can provide good conditions for studying the origin and acceleration propagation of cosmic rays. 

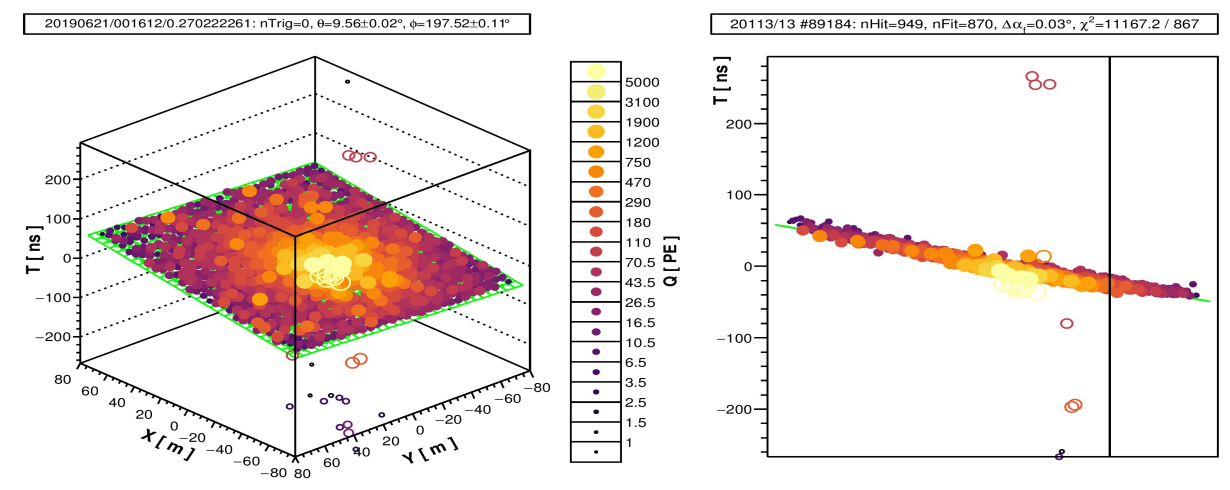

Figure 2: An event in pool 1 of WCDA.

The WCDA array is $78000 \mathrm{~m}^{2}$ which composed of 3 pools, and pool 1 and pool 2 are both $150 \mathrm{~m} \times 150 \mathrm{~m}$, the third pool array is $300 \mathrm{~m} \times 110 \mathrm{~m}$. The effective observed energy range of WC$\mathrm{DA}$ is $100 \mathrm{GeV}-30 \mathrm{TeV}$. There are 3120 detector units in total at 3 pools. Therein pool 1 installation has been completed now and there are 900 detector units in all at pool 1 . The 900 detector units divide into 25 clusters and each cluster is comprised of 4 groups in which one group is made up of 9 detector units. One electronics board control 9 detectors units that are one group. Each detector unit is $5 \mathrm{~m} \times 5 \mathrm{~m}$ that includes one 1.5 inches PMT and one 8 inches PMT. The 8 inches PMT direction of the cable is put north by east $37.5^{\circ}$ which the gain is middle of this direction because of the geomagnetic effects.

\section{Monte Carlo simulation of moon shadow}

The propagation of the primary cosmic rays between the moon and earth is carefully studied by using the method of Monte Carlo simulation. The simulation process of moon shadow can be divided into 3 steps: firstly the cosmic ray particle is traced back to the moon from the top of the atmosphere (about $40 \mathrm{~km}$ ) of the earth; then the simulated moon shadow events are going to the air shower cascading process; thirdly, it is to simulate the detector response to the secondary particle projected in the detector.

\subsection{The transportation in Geomagnetic Field (GMF)}

Here we just simulate the moon shadow signal and we regard it as a positive signal. In order to improve the efficiency of hitting the moon disk, we reverse the charge of the primary particle and track them backwards towards the Moon. Three possible results could be achieved by the particle. The first one is that the particle may reverse the direction and never hit the moon. The second one is that the particle may be trapped in the geomagnetic field. The third one is the particle hit the moon disk. Here just the third case is our valid MC moon shadow samples which will proceed in the next procedure. And once the particle hits the moon, the following event information about primary mass ID, energy, direction, MJD and so on are kept for the following simulation.

Since the magnetic field of the moon is far less than earth's, we can lose sight of the magnetic field near the moon and take into account the magnetic field near the earth. For the geomagnetic 
field, the International Geomagnetic Reference Field (IGRF) model [四] is used in this work. Figure $B$ is the geomagnetic field strength distribution at one earth radius. In the figure, latitude is on the $y$ axis, longitude is on the $\mathrm{x}$ axis, and the intensity of the magnetic field is expressed in $\mathrm{nT}$.

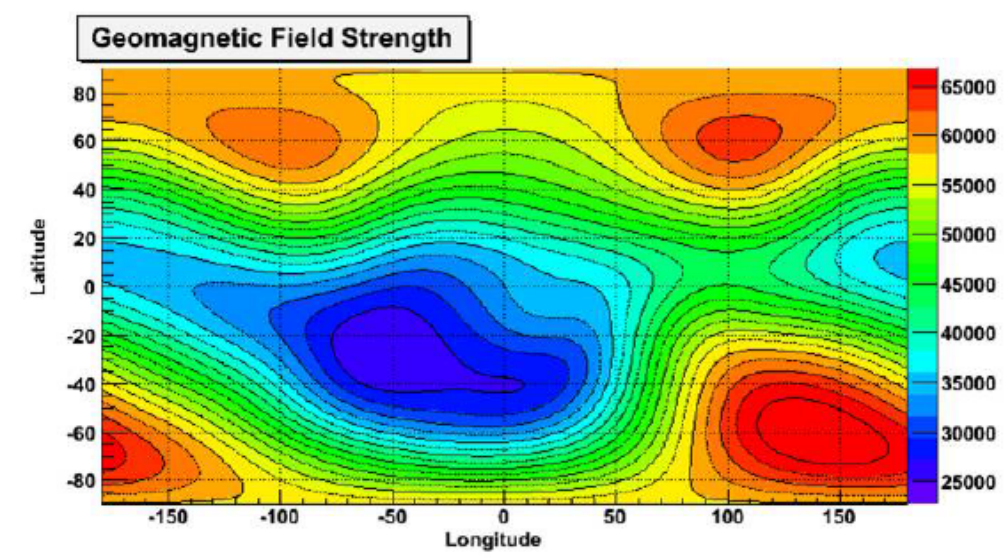

Figure 3: The strength from International Geomagnetic Reference Field model at one earth radius.

At this moment, just two kind of primary particles are simulated in this work: protons, helium nuclei. The proton energy range is from $100 \mathrm{GeV}$ to $100 \mathrm{TeV}$, the other is $1 \mathrm{TeV}-100 \mathrm{TeV}$. The direction of the simulated primary direction of cosmic rays is extracted along the moon orbit with certain opening angle. The detailed opening angle range is depending on the primary energy. The orbit of the moon, the left panel and the event direction, the right panel, is shown in Figure $\$$ w which is relative to the time is from October 1st, 2018 to October 31th 2018.
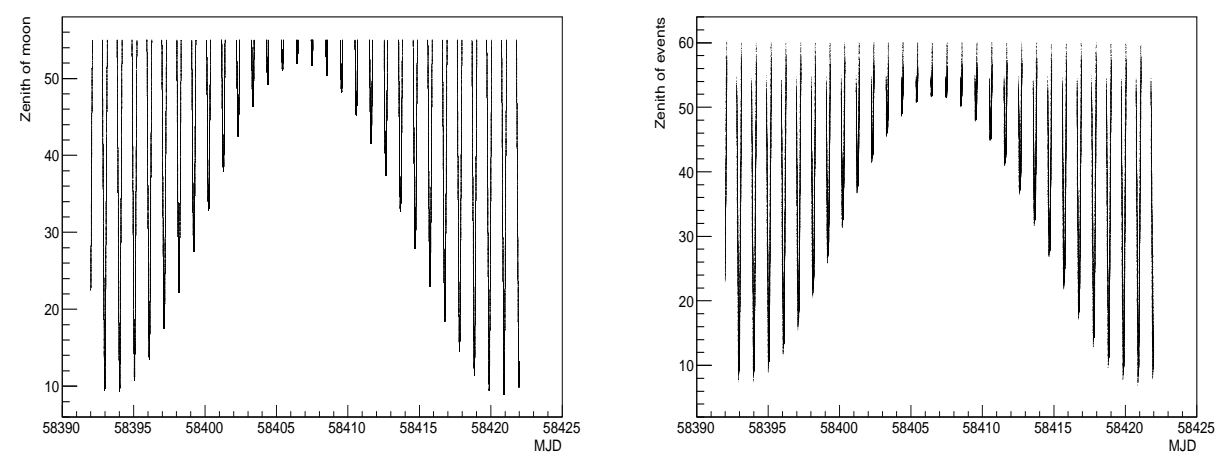

Figure 4: Left: the zenith angle distribution with mjd time for the moon orbit. Right: the zenith angle distribution of incident primary particles at the same time.

As we know, the relationship between the deflection angle and energy is: $\theta=\frac{1.6 Z}{E(T e V)}$, where $\mathrm{E}$ is the energy of cosmic ray and $\mathrm{Z}$ is the charge of the particle. The MC result is shown in Figure $\square$ that matches the calculation as we expected.

With a simple consideration of Point Spread Function (PFS) of the detector, a simulated moon shadow can be reproduced in Figure 6 . The plot in the left panel is the moon shadow in the ideal detector which means detector has perfect angular resolution; the plot in the middle is the moon 


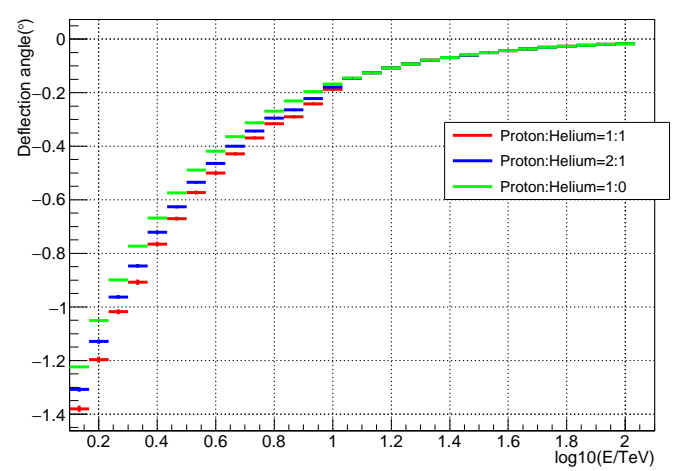

Figure 5: The relationship between deflection angle and energy.

shadow with PFS around 1.31 degree; the right panel is the smoothed result of the middle one with the smoothing radius approximately 1.31 degree. From this plot, one can see as we expected, the moon shadow has displacement in RA direction, no deflection in DEC direction. Thus we believe our MC procedure and result are solid.
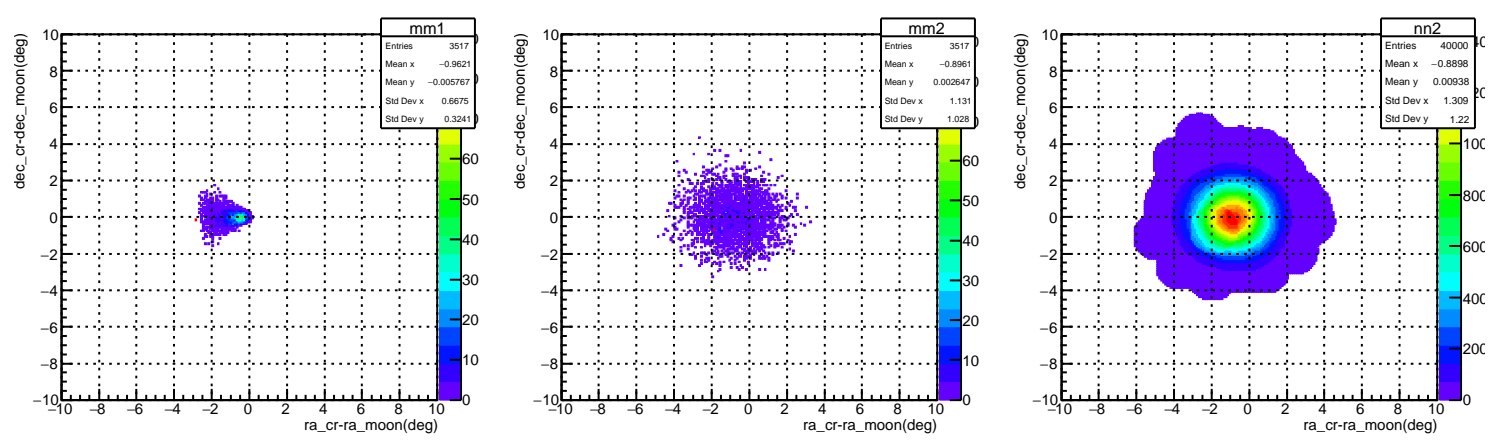

Figure 6: One simulated moon shadow example.

\subsection{Air shower cascade and detector response simulation}

The CORSIKA [5] program package is used to simulate the process of Extensive Air Shower (EAS) in the atmosphere, the hadronic model EPOS-LHC and Fluka are used in this work. After the EAS, we should take into account the response to secondary particles in the detector. The so-called G4WCDA programme which is based on the Geant4 [6] is used to consider the detector geometry, particle interaction with materials inside WCDA detectors. And the project area of randomly sampled is within $300 \mathrm{~m} \times 300 \mathrm{~m}$ around the pool 1 center. There are simulation details as shown in Table $\mathbb{U}$.

\section{Displacement of moon shadow in west-east direction from data}

The data used in this result is selected from April 1st, 2019 to June 18th, 2019. The standard reconstruction procedure has been adopted, all reconstruction data with the zenith angle less than 
Table 1: The simulation detailed conditions.

\begin{tabular}{ccccccc}
\hline Primary & $\begin{array}{c}\text { energy } \\
(\mathrm{TeV})\end{array}$ & $\Omega^{\circ}$ & $\begin{array}{c}\text { GMF } \\
\text { all events }\end{array}$ & $\begin{array}{c}\text { GMF } \\
\text { moon shadow events }\end{array}$ & Corsika & Geant4 \\
\hline Proton & $0.1-0.7$ & 20 & $1.2 \times 10^{8}$ & $2.0 \times 10^{4}$ & $2.0 \times 10^{4}$ & $2.0 \times 10^{4}$ \\
Proton & $0.7-1$ & 5 & $2.2 \times 10^{7}$ & $6.0 \times 10^{4}$ & $6.0 \times 10^{4}$ & $6.0 \times 10^{4}$ \\
Proton & $1-10$ & 3 & $6.2 \times 10^{6}$ & $4.7 \times 10^{4}$ & $4.7 \times 10^{4}$ & $4.7 \times 10^{4}$ \\
Proton & $10-100$ & 1 & $7.3 \times 10^{4}$ & $5.0 \times 10^{3}$ & $5.0 \times 10^{3}$ & $5.0 \times 10^{3}$ \\
Helium & $1-10$ & 5 & $2.4 \times 10^{6}$ & $6.6 \times 10^{3}$ & $6.6 \times 10^{3}$ & $6.6 \times 10^{3}$ \\
Helium & $10-100$ & 1 & $3.6 \times 10^{4}$ & $2.5 \times 10^{3}$ & $2.5 \times 10^{3}$ & $2.5 \times 10^{3}$ \\
\hline
\end{tabular}

45 degree is used. In the moon shadow analysis, to estimate the background is a key point. Here the equal zenith angle method is used, the details about this method can be found in [ $[\mathrm{U}]$.

The Figure $\square$ shows the preliminary result on moon shadow observation, the significance map of moon shadow in different nfit range, where nfit is a parameter to characterize the event energy. One can find that the moon shadow size turns smaller when the nfit becomes higher, it is reasonable. And the displacement along RA direction turns smaller with the increase of nfit.
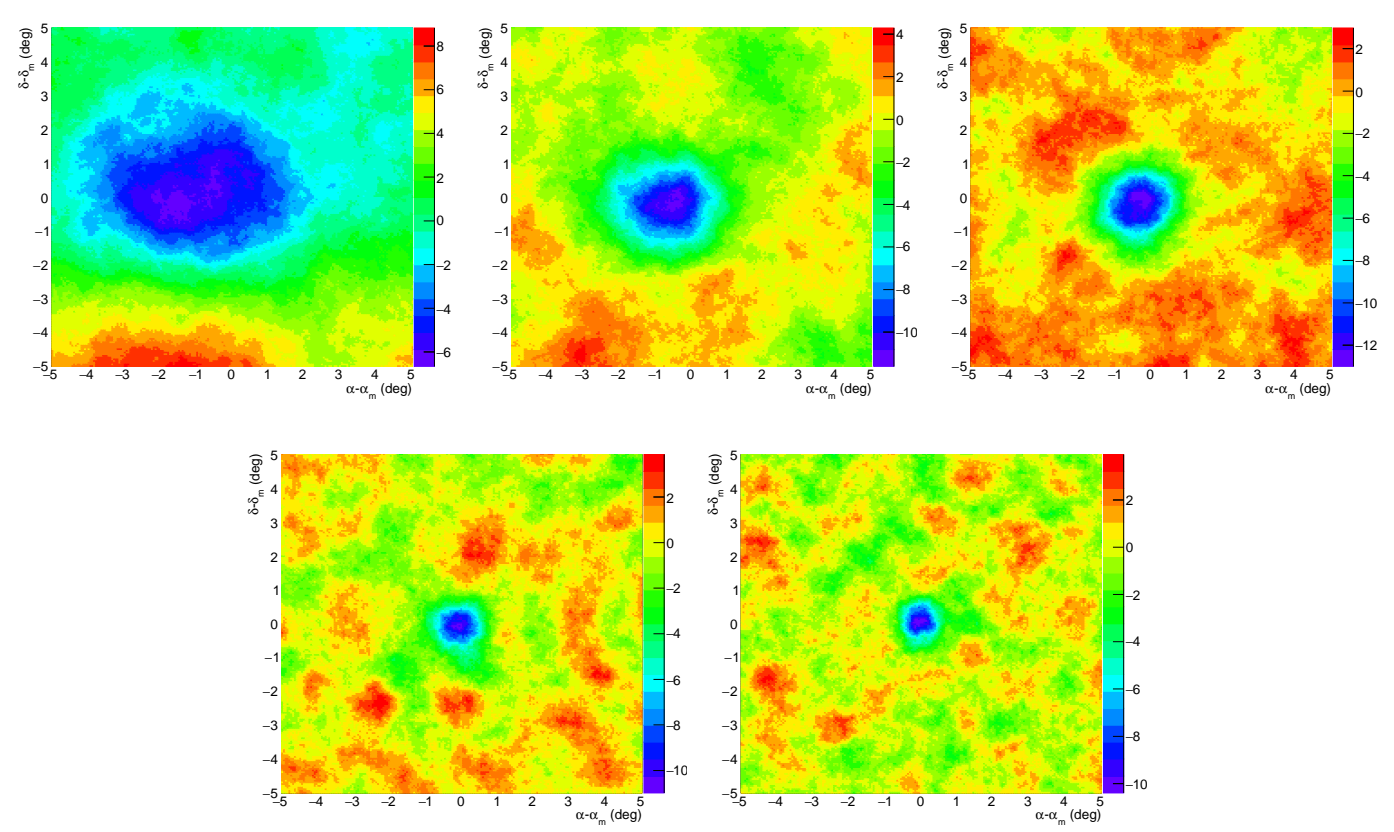

Figure 7: WCDA data moon shadow significance map observedd in 5 nfit range: 20-50,50-100,100200,200-300,300-900.

The displacement of moon shadow along RA direction is compared with MC result and shown in Figure 8. We can see the error bar is big when MC sample is in low nfit range in which the sample is less. One can see a good agreement can be getting between data and MC within the error bar. 


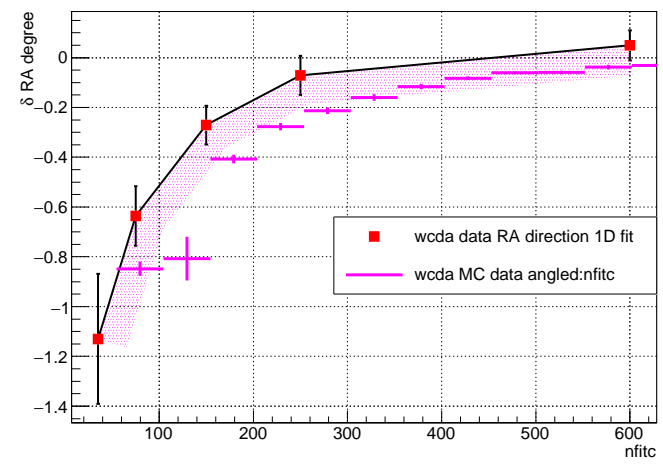

Figure 8: The displacement of the moon shadow in RA direction as function of nfit, the MC result is also shown in pink line.

\section{Conclusion and outlook}

In this work, the details about the moon shadow simulation are introduced. Some preliminary results are shown and preliminary comparisons between data and $\mathrm{MC}$ are done, within error the data and MC showed good agreement.

As discussed in the text different primary particles will lead to different effects. To reduce the contamination from heavier components, such as $\mathrm{C}-\mathrm{N}-\mathrm{O}, \mathrm{Mg}-\mathrm{Al}-\mathrm{Si}$ and Iron species should be carefully studied shown in Figure $Q$. And at the same time to disentangle the charge dependence on

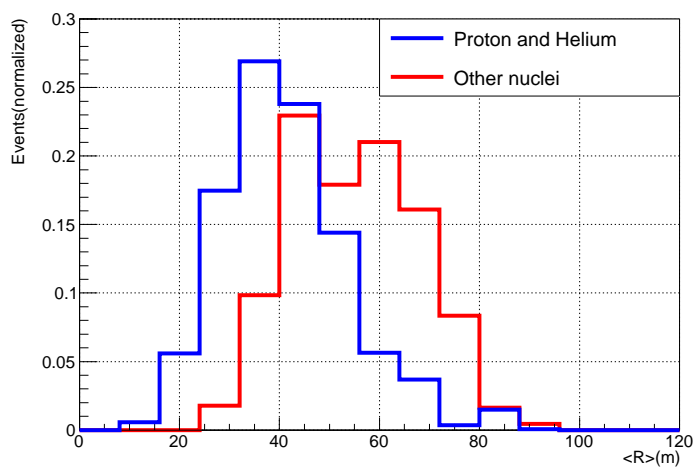

Figure 9: The average transverse extension radius distribution of proton and helium.

energy determination is also a very important topic, and the effect from different hadronic models is also a problem to be estimated. All these studies are main stuff in the future work.

\section{Acknowledgments}

This work is supported by the National Key R\&D programme of China (No.2018YFA0404201) and supported in China by NSFC (11675187), the Chinese Academy of Sciences, the Key Laboratory of Particle Astrophysics, CAS. 


\section{References}

[1] On behalf of the LHAASO collaboration, Acta Astronomica Sinica, 2019, 60(3):1-16.

[2] The ARGO-YBJ collaboration, Astroparticle Physics, 2017, 90:20-27.

[3] Alexandreas D E, Allen R C, Berley D, et al., Physical Review D, 1991, 43(5):1735-1738.

[4] Bo Wang. Ph,D.Thesis, Beijing: Institute of High Energy Physics Chinese Academy of Sciences, 2006.

[5] Heck D et al., Report FZKA, 1998, 6019.

[6] Geant4 User's Guide: http://geant4.web.cern.ch/geant4.

[7] The Tibet AS $\gamma$ Collaboration, The Astrophysical Journal, 2005, 633:1005-1012. 